\title{
Fuzzy Rule based Enhanced Homomorphic Encryption in Cloud Computing
}

\author{
Randeep Kaur \\ Department of Computer Science and \\ Engineering, SGGSWU, Fatehgarh Sahib, India, \\ Punjab (140406)
}

\author{
Supriya Kinger \\ Department of Computer Science and \\ Engineering, SGGSWU, Fatehgarh Sahib, \\ India,Punjab Punjab (140406)
}

\begin{abstract}
Now a day's internet services are one of the most popular services for transferring data and information. Cloud Computing is also based on internet services used for storing, transferring and many more operations related to data \& work. As it is based on internet services, security is one of the major concerns in this. Numbers of methods or algorithms were implemented for securing data and information. And when the data or information is to be used it has to be decrypted so that it could be used for further process. This becomes a major concern as time consumption increased. So, to solve this issue a new fully homomorphic environment has been introduces in cloud computing. This resolves the time consumption issue but introduce a new issue that is storage space consumption. This work is introduces a new enhanced fuzzy based fully homomorphic environment. As fuzzy is a artificial intelligent system, here it is rule based intelligent system which work on 27 defined rules and helps to achieve this objective of reduces space consumption issue by using lossless compression. Comparative analysis can be done \& proves that this enhanced environment is very secure and reduces burden of the network as well.
\end{abstract}

\section{Keywords}

Cloud computing, Security, Homomorphic Encryption, Fuzzy rules.

\section{INTRODUCTION}

Cloud computing is becoming very popular computing paradigm for network applications in open distributed environments. In essence, the idea is to host various application servers in a virtual network environment ("cloud") and offer their use through the concept of (Web) and other services. Contrary to classical network applications approach in the form of client-server model, in a cloud environment users do not access individual application servers, do not establish direct connections with them, do not send request messages directly to those servers, and do not receive direct replies from them. Instead, clients access those application servers through cloud access proxies, special servers that perform publishing and exporting various (usually Web) services available in a cloud. In these environment compared to client-server, classical network security have an important role. Not only this security services i.e. authentication, authorization, confidentially, integrity, authorization, etc must be provided to the client in a transparent way and the environment which comprises of delegated authorities and distributed component [1]. There are still many open and interesting issues regarding cloud computing paradigm and standards are still evolving. But, it is a general opinion that security is indeed one of the most important issues. In the recent IDC report over $74 \%$ of users think that security is dominant issue for widespread use of cloud computing services:

There are a lot of security issues in cloud computing service environments such as virtualization, distributes big data processing, serviceability, traffic-handling, application security, access control, authentication, cryptography and etc. Especially, data access using various resources needs user authentication and access control model for integrated management and control in cloud computing environments. Cloud computing security is a hot topic for research, its freshness, interestingness and recognition created an appeal for researches to pursue this topic in specific. Many security concerns evolved while weighing the benefits of using cloud computing over local resources.

\section{FULLY HOMOMORPHIC ENCRYPTION}

Homomorphic encryption is a type of encryption that allow computation of specific type that carries out cipher text give an encrypted results which on decryption matches the results of the operation done on the plaintext.

For modern communication this is the desirable feature. Homomorphic encryption allows chaining for different services together keeping the data transparent to each of those services, for example the chain of different services from various companies like 1)the currency exchange rate 2)shipping 3)calculate the tax, on transaction without showing the unencrypted data to each of the serves. Retrieval schemes - private information, secured systems of Homomorphic encryption is a type of encryption that allow computation of specific type that carries out cipher text give an encrypted results which on decryption matches the results of the operation done on the plaintext.

For modern communication this is the desirable feature. Homomorphic encryption allows chaining for different services together keeping the data transparent to each of those services, for example the chain of different services from various companies like 1)the currency exchange rate 2)shipping 3)calculate the tax, on transaction without showing the unencrypted data to each of the serves. Retrieval schemes - private information, secured systems of voting [2], hash functions which are collision resistant and using cloud computing widespread to ensure processed data confidentiality can be produced by cryptosystems using their homomorphic property.

There are a number of less efficient but fully homomorphic and some efficient but cryptosystems that are partially homomorphic. Although an unintentional homomorphic could be projected to attacks on this grounds, but if carefully treated 
computations can be performed securely using homomorphism.

All the examples discussed above allow only one operation of homomorphic computation [either multiplication or addition] on plaintexts. Cryptosystem which allows both multiplication and addition [plaintexts ring structure is preserved] is called as (FHE) fully homomorhic encryption and is quiet powerful. When we use a scheme like this evaluation of the circuit can be done homomorphically, allowing making of programs that will follow their encryption inputs to get their output encryption .As inputs can never be decrypted by such a program, it can be used by a party which is entrusted without displaying its internal state and input. Cryptosystem -fully and efficient homomorphic existence of such would have great effects in private computations outsourcing for example in relation to cloud computing. In [FHE] homomorphic part can be explained in the form of category theory. If category is $\mathrm{C}$ and integers are whose objects [e.g. data of finite streams] and multiplication and addition are included in whose morphisms, then encryption process of [FHE] is C's end function. Approach called categorical approach gives way for generalization apart from ring structure [multiplication and addition finitely composition] of integer. If morphisms of some C's super category include all functions that are computable or functions recursive which are primitive, then encryption that passes for super category as end factor is homomorphic more fully as in encrypted data extra operations are possible [ex loops \& conditionals]

Encryption of fully homomorphic utility is recognized for very long. After producing RSA, in a year of its production problem of producing above scheme was produced first. [3]. whether $[\mathrm{FHE}]$ is possible was unclear for greater than 30 years. In this time period best answer was encryption of Boneh-Goh-Nissim which does uncountable times of addition operations \& one multiplication at the most.

\section{PROPOSED FUZZY RULE BASED HOMOMORPHIC ENCRYPTION}

The first approach to artificial intelligence were (crisp) expert systems and models imitating the functionality of the human brain- so called artificial neural networks (ANNs). While conventional expert systems are rule based systems, which manipulate symbolic expressions based on traditional binary logic, neural nets are systems with, in most cases, real valued input and output, which can learn from sample data, but without the possibility to survey the actions inside. Fuzzy systems are rule based systems which are able to process vague, imprecise data. In this sense, fuzzy systems can be regarded as generalized rule based expert systems. This generalization requires a mathematical formulation of impreciseness and inference methods adapted to this model.

To enhance the FHE based system fuzzy rule based system is being used by the generated cloud environment. This fuzzy rule based system can be worked on 27 different if-then rules and by using this cloud system will perform better and reduce the burden of the network. These rules are standard rules prepared by fuzzy rule based system and this local cloud system follows these rules and performs their actions. The proposed work is to implement Fully Homomorphic encryption environment by using .NET Environment and also to simulate a new fuzzy rule based FHE system which reduce the heavy burden of network and storage.

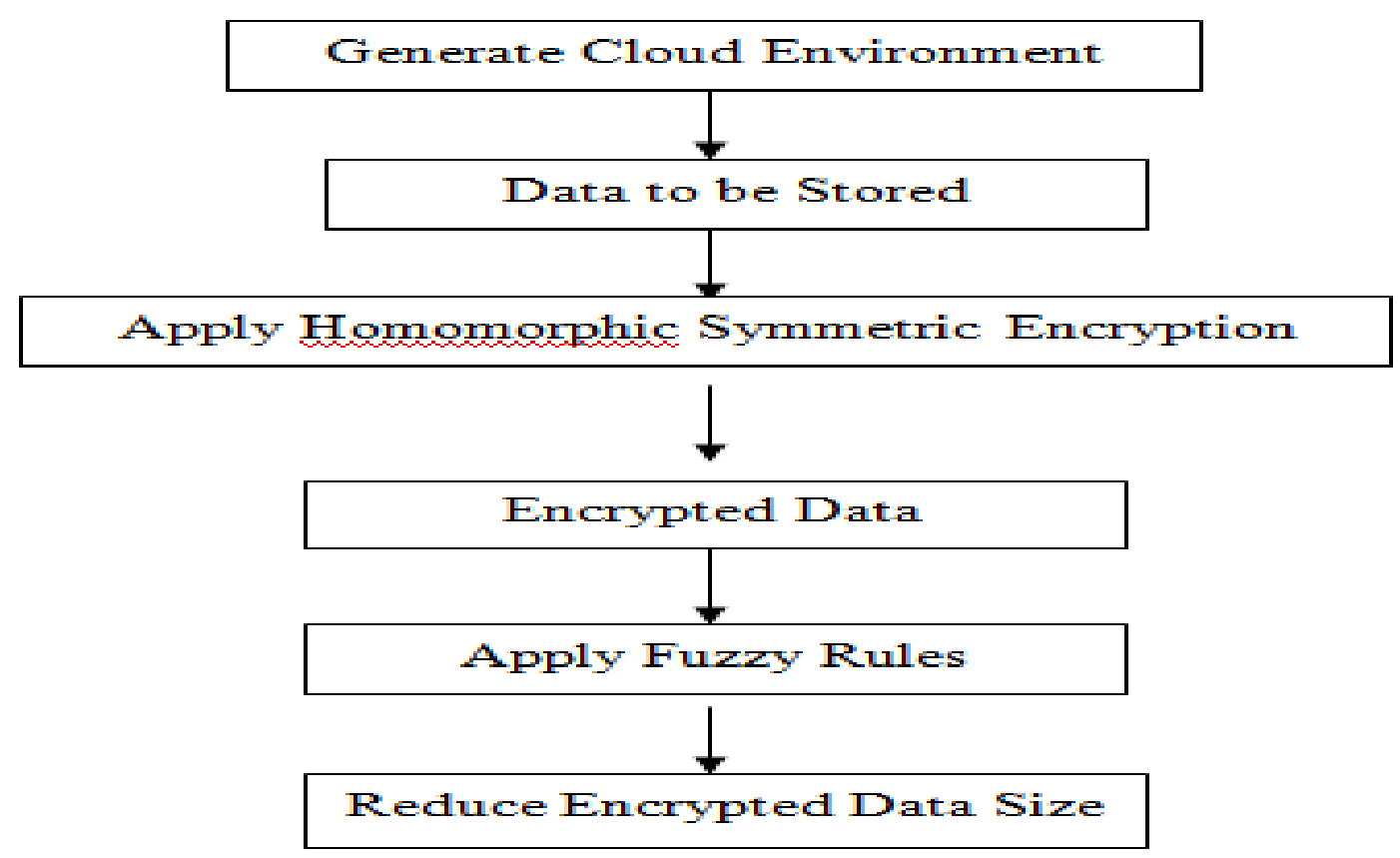

Fig 1: Flow of the work

In this work, a local client server environment is generated to implement local cloud environment. This environment provides users to access the cloud with login id's and passwords to use it for further storage of data. The data can be stored in encrypted form by using encryption algorithm then analysis can be done to analyze the performance of fully homomorphic encryption by taking an example of merging operation. This homomorphic encryption provides 
environment that data can be used in encrypted form but a new issue of high burden on network can be introduced while using it. So, this work is to reduce the burden by using fuzzy if then rules which will define in the next sections. This fuzzy based rule helps to reduce the encryption data size while they used as in encrypted form as well.

The step wise algorithm design is as follows:

Step $1 \rightarrow$ To use this cloud environment user has to create their account as to get authentication and can login only by using this information.

Step $2 \rightarrow$ Once a user can login different action will be perform by him that are upload data, merge operation, check resultant graphs.

Step $3 \rightarrow$ When user uploads data, some information related to data uploaded can be filled out and then data could be saved in the encrypted form by using RSA encryption algorithm.

Step $4 \rightarrow$ To implement the action of fully homomorphic environment, this system works on an example of merging operation.

Step $5 \rightarrow$ Merging will be done by using three methods that are 'download \& merge', 'FHE' and 'Fuzzy based 'FHE'.

Step $6 \rightarrow$ Comparative analysis of the system is on the basis of all above method being done after all operations.

\section{FUZZY RULES}

\begin{tabular}{|c|c|}
\hline $\begin{array}{l}\quad \checkmark \quad \text { Rule=1 } \\
\text { If } \\
\text { File } 1==\text { File 1_FHE } \\
\text { File } 2==\text { File 2_FHE } \\
\text { File } 3==\text { File 3_FHE } \\
\text { Then } \\
\text { Perform no action. }\end{array}$ & $\begin{array}{l}\quad \checkmark \quad \text { Rule }=\mathbf{3} \\
\text { If } \\
\text { File } 1==\text { File } 1 \text { _FHE } \\
\text { File } 2==\text { File 2_FHE } \\
\text { File } 3>\text { File 3_FHE } \\
\quad \begin{array}{c}\text { Then } \\
\text { Perform no action }\end{array}\end{array}$ \\
\hline $\begin{array}{l}\text { If } \checkmark \text { Rule = 2 } \\
\text { File } 1==\text { File 1_FHE } \\
\text { File } 2==\text { File 2_FHE } \\
\text { File } 3<\text { File 3_FHE } \\
\text { Then } \\
\text { Check size of file } 3 \\
\text { (File3_FHE) } \\
\text { Compare }- \text { How much greater. } \\
\text { Then Compress that File using } \\
\text { Loss less compression } \\
\text { technique. } \\
\text { By using Loss less } \\
\text { compression technique File } \\
\text { data no loss. But it reduces the } \\
\text { file size. }\end{array}$ & $\begin{array}{l}\quad \checkmark \quad \text { Rule }=\mathbf{4} \\
\text { If } \\
\text { File } 1==\text { File 1_FHE } \\
\text { File } 2<\text { File 2_FHE } \\
\text { File } 3=\text { = File 3_FHE } \\
\text { Then } \\
\text { Perform no action. }\end{array}$ \\
\hline $\begin{array}{l}\quad \checkmark \quad \text { Rule }=\mathbf{5} \\
\text { If } \\
\text { File } 1==\text { File 1_FHE } \\
\text { File } 2<\text { File 2_FHE } \\
\text { File } 3>\text { File 3_FHE } \\
\text { Then } \\
\text { Perform no action }\end{array}$ & $\begin{array}{l}\quad \checkmark \quad \text { Rule }=\mathbf{1 1} \\
\text { If } \\
\text { File } 1<\text { File 1_FHE } \\
\text { File } 2<\text { File 2_FHE } \\
\text { File } 3=\text { = File 3_FHE } \\
\text { Then } \\
\text { Perform No action. } \\
\end{array}$ \\
\hline $\begin{array}{l}\quad \checkmark \quad \text { Rule }=\mathbf{6} \\
\text { If } \\
\text { File } 1<\text { File 1_FHE } \\
\text { File } 2<\text { File 2_FHE } \\
\text { File } 3>\text { File 3_FHE }\end{array}$ & $\begin{array}{l}\quad \checkmark \quad \text { Rule }=\mathbf{1 2} \\
\text { If } \\
\text { File } 1<\text { File 1_FHE } \\
\text { File } 2==\text { File 2_FHE } \\
\text { File } 3>\text { File 3_FHE }\end{array}$ \\
\hline
\end{tabular}

\begin{tabular}{|c|c|}
\hline $\begin{array}{l}\text { Then } \\
\text { Perform No action. }\end{array}$ & $\begin{array}{l}\text { Then } \\
\text { Perform No action. }\end{array}$ \\
\hline $\begin{array}{l}\quad \checkmark \quad \text { Rule }=7 \\
\text { If } \\
\text { File } 1==\text { File 1_FHE } \\
\text { File } 2>\text { File 2_FHE } \\
\text { File } 3==\text { File 3_FHE } \\
\text { Then } \\
\text { Perform no action. }\end{array}$ & $\begin{array}{l}\text { If } \quad \text { Rule }=\mathbf{1 3} \\
\text { File } 1==\text { File 1_FHE } \\
\text { File } 2>\text { File 2_FHE } \\
\text { File } 3<\text { File 3_FHE } \\
\text { Then } \\
\text { Check size of file } 3 \\
\text { (File3_FHE) } \\
\text { Compare - How much } \\
\text { greater. } \\
\text { Then Compress that File } \\
\text { using Loss less compression } \\
\text { technique. } \\
\text { By using Loss less } \\
\text { compression technique File } \\
\text { data no loss. But it reduces } \\
\text { the file size. }\end{array}$ \\
\hline $\begin{array}{l}\quad \checkmark \quad \text { Rule = 8 } \\
\text { If } \\
\text { File } 1==\text { File 1_FHE } \\
\text { File } 2<\text { File 2_FHE } \\
\text { File } 3<\text { File 3_FHE } \\
\text { Then } \\
\text { Check size of file } 3 \\
\text { (File3_FHE) } \\
\text { Compare - How much greater. } \\
\text { Then Compress that File using } \\
\text { Loss less compression } \\
\text { technique. } \\
\text { By using Loss less } \\
\text { compression technique File } \\
\text { data no loss. But it reduces the } \\
\text { file size. }\end{array}$ & $\begin{array}{l}\text { If } \quad \checkmark \text { Rule = 14 } \\
\text { File } 1<\text { File 1_FHE } \\
\text { File } 2<\text { File 2_FHE } \\
\text { File 3< File 3_FHE } \\
\text { Then } \\
\text { Check size of file } 3 \\
\text { (File3_FHE) } \\
\text { Compare - How much } \\
\text { greater. } \\
\text { Then Compress that File } \\
\text { using Loss less compression } \\
\text { technique. } \\
\text { By using Loss less } \\
\text { compression technique File } \\
\text { data no loss. But it reduces } \\
\text { the file size. }\end{array}$ \\
\hline $\begin{array}{l}\quad \quad \checkmark \quad \text { Rule }=9 \\
\text { If } \\
\text { File } 1==\text { File 1_FHE } \\
\text { File } 2>\text { File 2_FHE } \\
\text { File } 3>\text { File 3_FHE } \\
\text { Then } \\
\text { Perform No action }\end{array}$ & $\begin{array}{l}\quad \checkmark \quad \text { Rule }=\mathbf{1 5} \\
\text { If } \\
\text { File } 1>\text { File 1_FHE } \\
\text { File } 2==\text { File 2_FHE } \\
\text { File } 3==\text { File 3_FHE } \\
\text { Then } \\
\text { Perform No action. }\end{array}$ \\
\hline $\begin{array}{l}\quad \checkmark \quad \text { Rule }=\mathbf{1 0} \\
\text { If } \\
\text { File } 1<\text { File 1_FHE } \\
\text { File } 2==\text { File 2_FHE } \\
\text { File } 3==\text { File 3_FHE } \\
\text { Then } \\
\text { Perform no action. }\end{array}$ & $\begin{array}{l}\quad \checkmark \quad \text { Rule }=\mathbf{1 6} \\
\text { If } \\
\text { File } 1>\text { File 1_FHE } \\
\text { File } 2>\text { File 2_FHE } \\
\text { File } 3>\text { File 3_FHE } \\
\text { Then } \\
\text { Perform No action. }\end{array}$ \\
\hline $\begin{array}{l}\quad \checkmark \quad \text { Rule }=\mathbf{1 7} \\
\text { If } \\
\text { File } 1>\text { File 1_FHE } \\
\text { File } 2<\text { File 2_FHE } \\
\text { File } 3==\text { File 3_FHE } \\
\text { Then } \\
\text { Perform No action. }\end{array}$ & $\begin{array}{l}\quad \checkmark \quad \text { Rule }=\mathbf{2 2} \\
\text { If } \\
\text { File } 1<\text { File 1_FHE } \\
\text { File } 2>\text { File 2_FHE } \\
\text { File } 3>\text { File 3_FHE } \\
\text { Then } \\
\text { Perform No action. }\end{array}$ \\
\hline $\begin{array}{l}\quad \checkmark \quad \text { Rule }=\mathbf{1 8} \\
\text { If } \\
\text { File } 1<\text { File 1_FHE } \\
\text { File } 2==\text { File 2_FHE } \\
\text { File 3< } \\
\text { Then } \\
\text { Check sile } 3 \text { size of file }\end{array}$ & $\begin{array}{l}\quad \checkmark \quad \text { Rule }=\mathbf{2 3} \\
\text { If } \\
\text { File } 1<\text { File 1_FHE } \\
\text { File } 2>\text { File 2_FHE } \\
\text { File } 3<\text { File 3_FHE } \\
\text { Then } \\
\text { Check size of file }\end{array}$ \\
\hline
\end{tabular}




\begin{tabular}{|c|c|}
\hline $\begin{array}{l}\text { (File3_FHE) } \\
\text { Compare - How much greater. } \\
\text { Then Compress that File using } \\
\text { Loss less compression } \\
\text { technique. } \\
\text { By using Loss less } \\
\text { compression technique File } \\
\text { data no loss. But it reduces the } \\
\text { file size. }\end{array}$ & $\begin{array}{l}\text { (File3_FHE) } \\
\text { Compare - How much } \\
\text { greater. } \\
\text { Then Compress that File } \\
\text { using Loss less compression } \\
\text { technique. } \\
\text { By using Loss less } \\
\text { compression technique File } \\
\text { data no loss. But it reduces } \\
\text { the file size. }\end{array}$ \\
\hline $\begin{array}{l}\quad \checkmark \quad \text { Rule = 19 } \\
\text { If } \\
\text { File } 1>\text { File 1_FHE } \\
\text { File } 2>\text { File 2_FHE } \\
\text { File } 3<\text { File 3_FHE } \\
\text { Then } \\
\text { Check size of file } 3 \\
\text { (File3_FHE) } \\
\text { Compare - How much greater. } \\
\text { Then Compress that File using } \\
\text { Loss less compression } \\
\text { technique. } \\
\text { By using Loss less } \\
\text { compression technique File } \\
\text { data no loss. But it reduces the } \\
\text { file size. }\end{array}$ & $\begin{array}{l}\quad \checkmark \quad \text { Rule }=\mathbf{2 4} \\
\text { If } \\
\text { File } 1>\text { File 1_FHE } \\
\text { File } 2==\text { File 2_FHE } \\
\text { File } 3>\text { File 3_FHE } \\
\text { Then } \\
\text { Perform No action. }\end{array}$ \\
\hline $\begin{array}{l}\quad \checkmark \quad \text { Rule }=\mathbf{2 0} \\
\text { If } \\
\text { File } 1>\text { File 1_FHE } \\
\text { File } 2>\text { File 2_FHE } \\
\text { File } 3==\text { File 3_FHE } \\
\text { Then } \\
\text { Perform No action }\end{array}$ & $\begin{array}{l}\quad \checkmark \quad \text { Rule }=\mathbf{2 5} \\
\text { If } \\
\text { File } 1>\text { File 1_FHE } \\
\text { File } 2=\text { =File 2_FHE } \\
\text { File } 3>\text { File 3_FHE } \\
\text { Then } \\
\text { Perform No action }\end{array}$ \\
\hline $\begin{array}{l}\quad \checkmark \quad \text { Rule }=\mathbf{2 1} \\
\text { If } \\
\text { File } 1<\text { File 1_FHE } \\
\text { File } 2>\text { File 2_FHE } \\
\text { File } 3==\text { File 3_FHE } \\
\text { Then } \\
\text { Perform No action. }\end{array}$ & \\
\hline $\begin{array}{l}\quad \checkmark \quad \text { Rule = 26 } \\
\text { If } \\
\text { File } 1>\text { File 1_FHE } \\
\text { File } 2<\text { File 2_FHE } \\
\text { File } 3<\text { File 3_FHE } \\
\text { Then } \\
\text { Check size of file } 3 \\
\text { (File3_FHE) } \\
\text { Compare - How much greater. } \\
\text { Then Compress that File using } \\
\text { Loss less compression } \\
\text { technique. } \\
\text { By using Loss less } \\
\text { compression technique File } \\
\text { data no loss. But it reduces the } \\
\text { file size. }\end{array}$ & $\begin{array}{l}\text { If } \quad \text { Rule }=\mathbf{2 7} \\
\text { File } 1>\text { File 1_FHE } \\
\text { File } 2==\text { File 2_FHE } \\
\text { File } 3<\text { File 3_FHE } \\
\text { Then } \\
\text { Check size of file } 3 \\
\text { (File3_FHE) } \\
\text { Compare - How much } \\
\text { greater. } \\
\text { Then Compress that File } \\
\text { using Loss less compression } \\
\text { technique. } \\
\text { By using Loss less } \\
\text { compression technique File } \\
\text { data no loss. But it reduces } \\
\text { the file size }\end{array}$ \\
\hline
\end{tabular}

\section{RESULTS \& COMPARISON}

The performance of this proposed system can be analyzed by using three different scenarios which are explained in the next section. They are simple download and merge, fully homomorphic environment and fuzzy rule based cloud. The differences between these environments are:

- In the first download and merge environment, firstly files can be downloaded individually and then merge operation can be implemented on that. This is also a time consuming process because firstly files can be decrypted and then downloaded.

- In the second fully homomorphic encryption environment, no need to downloads the data or files. Files can be directly used in the encrypted form for merging operation. This will helps to reduce the time but because of encryption a heavy burden on the network will be generated by this.

- In the third fuzzy rule based FHE system, no need to download data as in FHE but in this different rules can be applied to the system which will affect the system to reduce time and network burden as well.

To analyze the performance of this cloud system, a comparative analysis can be done by using different methods. The comparative analysis of all these above method can be shown in a graphical manner in this cloud environment.

This comparative analysis for the .txt files as above defined methods are as shown in fig 2 .

\section{Graph}

Size

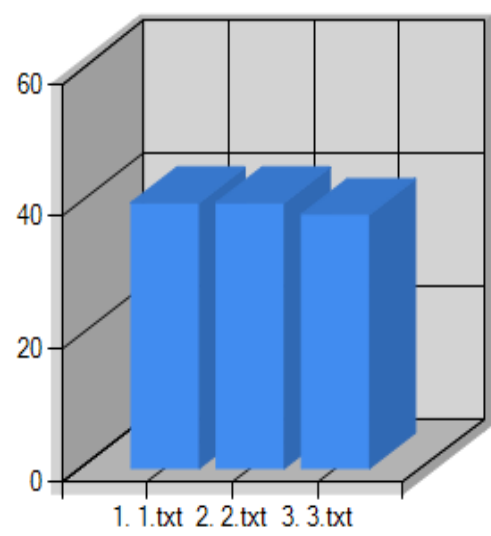

File Name

Fig 2: Comparative Analysis for .txt files

The comparative analysis of all these above method can be shown in a graphical manner in this cloud environment. This comparative analysis for the .pdf files as below defined methods are as shown in fig 3 . 


\section{Graph}

Size

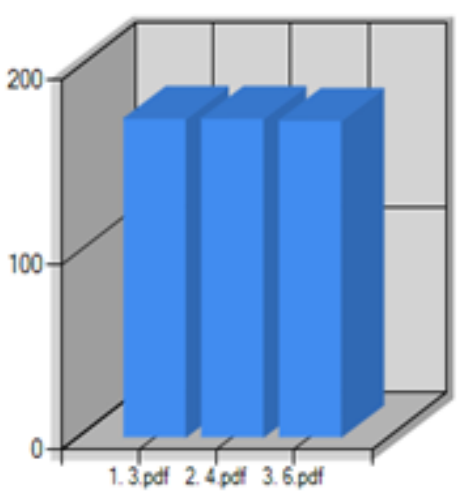

File Name

Fig 3: Comparative Analysis for .pdf files

The comparative analysis of all these above method can be shown in a graphical manner in this cloud environment. This comparative analysis for the .jpg files as above selected is as shown in fig 4.

\section{Graph}

Size

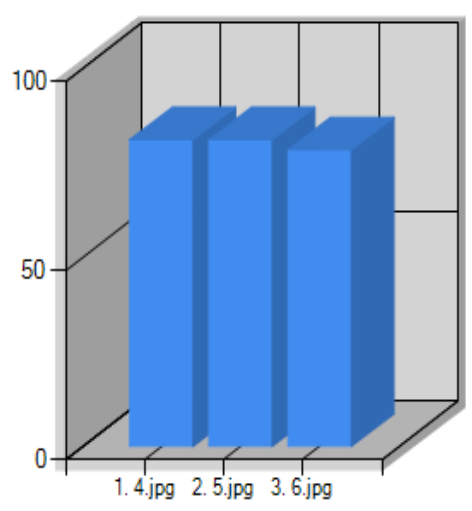

File Name

Fig 4: Comparative Analysis for .jpg files

The comparative analysis of all these above method can be shown in a graphical manner in this cloud environment. This comparative analysis for the .wav files as below defined methods are as shown in fig 5 .

\section{Graph}

Size

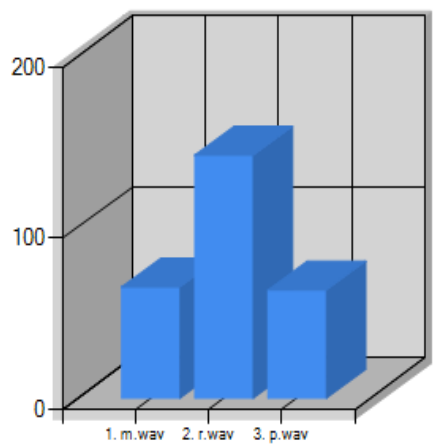

File Name

Fig 5: Comparative Analysis for .wav files

This work also presents the comparative analysis of all three methods for 5 files. The table 1 shows the file size for different files. The graph shown below in fig 6 represents that in 'FHE' method the size of all files are greater than their original size as calculate in 'Download \& Merge'. So, fuzzy based FHE is proposed as a rule based method which reduces the size of files using loseless compression and is work on the basis of some defined rules.

TABLE 1: File size while considering different files

\begin{tabular}{|l|l|l|l|l|l|}
\hline Files & 1 & 2 & 3 & 4 & 5 \\
\hline $\begin{array}{l}\text { Simple } \\
\text { Method }\end{array}$ & 32.1 & 45.15 & 68.6 & 49 & 81 \\
\hline FHE & 33.4 & 45.15 & 69.88 & 50 & 81 \\
\hline $\begin{array}{l}\text { Proposed } \\
\text { work }\end{array}$ & 31.6 & 43.12 & 66.4 & 47.4 & 78.8 \\
\hline
\end{tabular}

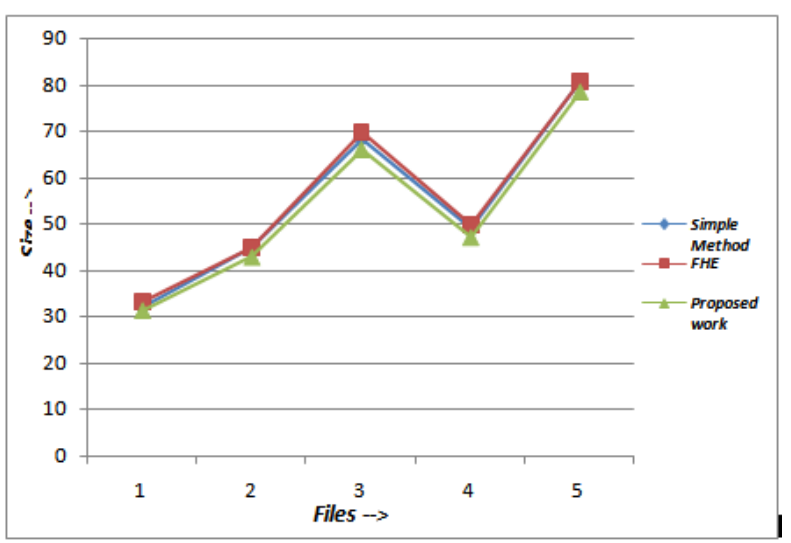

Fig 6: Comparative analysis using 5 files

The above graph represents comparison between the calculated size of the files by performing merging operation using three methods that are Simple download and merge method, Fully homomorphic encryption and fuzzy rule based FHE.(Fully Homomorphic Encryption). This shows that while 
fuzzy based fully homomorphic encryption is used then the file size get reduced and is less than other methods. This helps to reduce the burden on the network which is because of increasing size.

\section{CONCLUSION \& FUTURE WORK}

Security is a major concern in the cloud computing and there are number of algorithms and methods used in cloud computing to provide security and privacy. There is one big issue generated by implementing security using encryption algorithms that are user have to download data first and then it can be used for further processes. This process is a time consuming process. To solve this issue a fully homomorphic based encryption environment in cloud computing was introduce. Using this FHE user can use encrypted data as well without downloading that data. This will surely save time but as encrypted data is being used so data size gets increased. This will put a heavy burden on the network and also a cloud executor. So to resolve this issue this work introduces an intelligent system based on fuzzy. Fuzzy is here rule based system which creates some standard rules for this cloud environment. This rule based system use lossless compression algorithm to compress data without any loss and reduce the burden on the network. In this local cloud environment is generated and system is being tested using different type of data by taking an example of merging operation with 'download and merge', 'FHE' and 'Fuzzy rule based FHE'. Comparative analysis is being considered under this environment and results prove the efficiency of the new proposed fuzzy rule based FHE system.

As result analysis represents that proposed method work very well while tested on .txt, .jpg, .pdf and .wav files. For video files there is no testing undertaken. So, future work is to enhance this system by using more secure techniques and also to test the video data on the proposed system.

\section{REFERENCES}

[1] Ling Oian et al, "Cloud Computing: An overview" ,Ist International Conference, December,2009,pp.626-63.

[2] Ron Rivest, "Homomorphic Encryption" Volume 3, Issue ,October 29, 2002.

[3] R.L. Rivest, L. Adleman, and M. L. Dertouzos. On data banks and privacy homomorphisms. In Foundations of Secure Computation, 1978
[4] Bhabendu Kumar Mohanta and Debasis Gountia, "Fully homomorphic encryption equating to cloud security: An approach" IOSR Journal of Computer Engineering (IOSR-JCE), Volume 9, Issue 2 (Jan. - Feb. 2013), pp.46-50.

[5] Sunanda Ravindran and Parsi Kalpana, "Data Storage Security Using Partially Homomorphic Encryption in a Cloud" International Journal of Advanced Research in Computer Science and Software Engineering, Volume 3, Issue 4, April 2013, pp. 603-606.

[6] Dr. Chander Kant and Yogesh Sharma, "Enhanced Security Architecture for Cloud Data Security" International Journal of Advanced Research in Computer Science and Software Engineering, Volume 3, Issue 5, May 2013, pp. 571-575.

[7] Uma Somani, "Implementing Digital Signature with RSA Encryption Algorithm to Enhance the Data Security of Cloud in Cloud Computing," 2010 1st International Conference on Parallel, Distributed and Grid Computing (PDGC - 2010)

[8] RuWei Huang, Si Yu, Wei Zhuang and XiaoLin Gui, "Design of Privacy-Preserving Cloud Storage Framework" 2010 Ninth International Conference on Grid and Cloud Computing.

[9] Farzad Sabahi, "Cloud Computing Security Threats and Responses," IEEE Trans. on Cloud Computing., vol. 11, no. 6 , pp. $670\{684,2002$.

[10] Cong Wang, Qian Wang, Kui Ren and Wenjing Lou "Ensuring Data Storage Security in Cloud Computing." IEEE 2009.

[11] Mehmet Yildiz, Jemal Abawajy, Tuncay Ercan and Andrew Bernoth "A Layered Security Approach for Cloud Computing Infrastructure" 2009 10th International Symposium on Pervasive Systems, Algorithms, and Networks. @ 2009 IEEE.

[12] Sang-Ho Na, Jun-Young Park, Eui-Nam Huh "Personal Cloud Computing Security Framework" 2010 IEEE Asia-Pacific Services Computing Conference.

[13] Mohammed Achemlal, Sa"1d Gharout and Chrystel Gaber, "Trusted Platform Module as an Enabler for Security in Cloud Computing" Vol 978-1-4577-07377/11/\$26.00 @2011 IEEE. 